\title{
Transseptal Approach for Mitral Valve Replacement in Dextrocardia with Situs Inversus Totalis: A Case Report and Review of the Literature
}

\author{
Ovidiu Stiru, MD, PhD, Roxana Carmen Geana, MD, Razvan Radu Ilie, MD, Ovidiu Chioncel, MD, PhD, \\ Raluca Tulin MD, PhD, Liana Valeanu, MD, Serban Bubenek MD, PhD, Daniela Filipescu MD, PhD, \\ V. A. Iliescu, MD, PhD
}

Prof. Dr. C. C. Iliescu Emergency Institute for Cardiovascular Diseases, Bucharest, Romania

\section{ABSTRACT}

We consider mitral valve disease requiring surgery in a patient with dextrocardia and situs inversus totalis to be an exceptional finding. The transseptal approach for mitral valve surgery in dextrocardia represents a technical challenge owing to its anatomic particulars. We present the case of a 56-year-old female patient who had been diagnosed with situs inversus totalis in childhood and with chronic atrial fibrillation in adulthood and was under oral anticoagulant treatment. She was referred to our hospital for increasing dyspnea and palpitation. Transthoracic echocardiography detected severe mitral regurgitation associated with moderate tricuspid regurgitation, with normal left and right ventricular function. Contrast chest computed tomography (CT) and preoperative abdominal CT showed both dextrocardia and situs inversus totalis, with normal continuity of the inferior vena cava. Biatrial cannulation was performed with the surgeon standing on the right side of the patient, and mitral valve replacement using a transseptal approach was performed with the surgeon standing on the left side of the patient. In this case report, we emphasize the rarity of mitral valve disease in a patient with dextrocardia and the inherent potential difficulty that can appear in this particular anatomic condition.

\section{INTRODUCTION}

Dextrocardia is an anatomic particularity in which the heart is located in the middle of the mediastinum, with its base-to-apex axis directed to the right and caudal. Situs inversus totalis associated with dextrocardia has a rare incidence of 1 in 10,000 adult population [Garg 2009]. The concept of situs refers to the configuration of asymmetric organs in the human body. Anatomically, 3 types of situs have been described: situs solitus, which is the normal form of the placement of the organ in the human body; situs inversus, which is a mirror image of normal placement of the organs; and situs ambiguus. In situs inversus, anatomy and morphology

Received September 15, 2019; accepted December 12, 2019.

Correspondence: Ovidiu Stiru, MD, PbD, Prof. Dr. C. C. Iliescu Emergency Institute for Cardiovascular Diseases, Sos. Fundeni 258, sector 2, 022328 Bucharest, Romania; phone/fax: 400722207286 (e-mail: ovidiu_stiru@yahoo.com). of the abdominal and thoracic organ and vessel systems are conserved, the left atrium is located to the right, the left lung has 3 lobes and the right lung has 2 lobes, the stomach and the spleen are positioned on the right, and the larger lobe of the liver is on the left side of the body. In $50 \%$ to $80 \%$ of individuals with situs ambiguus, the abnormal arrangement of organ orientation early in fetal development results in severely disrupted cardiac development and function [Lowenthal 2019]. The causes of dextrocardia have not yet been identified, but it was observed that one quarter of the patients with this pathological condition have associated primary ciliary dyskinesia (Kartagener syndrome) with autosomal recessive inheritance [Zariwala 2013; Maldjian 2007]. Dextrocardia is not directly associated with race, ethnicity, or sex.

\section{CASE PRESENTATION}

A 56-year-old female patient, with hypertension (New York Heart Association Class III) and obesity as associated risk factors, was diagnosed with mitral valve disease associating severe regurgitation and mild stenosis 6 months before admission to our clinic. She was in atrial fibrillation on electrocardiography (ECG), and the preoperative chest X-ray

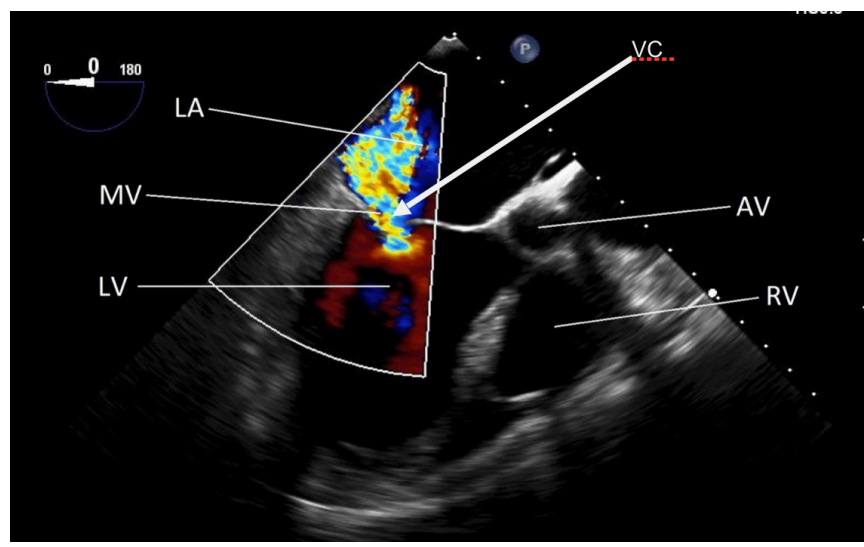

Figure 1. Transesophageal echocardiography 4-chamber view with color Doppler showing severe mitral valve regurgitation with eccentric regurgitant jet and a vena contracta width of $0.7 \mathrm{~cm}$. AV, aortic valve; LA, left atrium; LV, left ventricle; MV, mitral valve; RV, right ventricle; VC, vena contracta. 

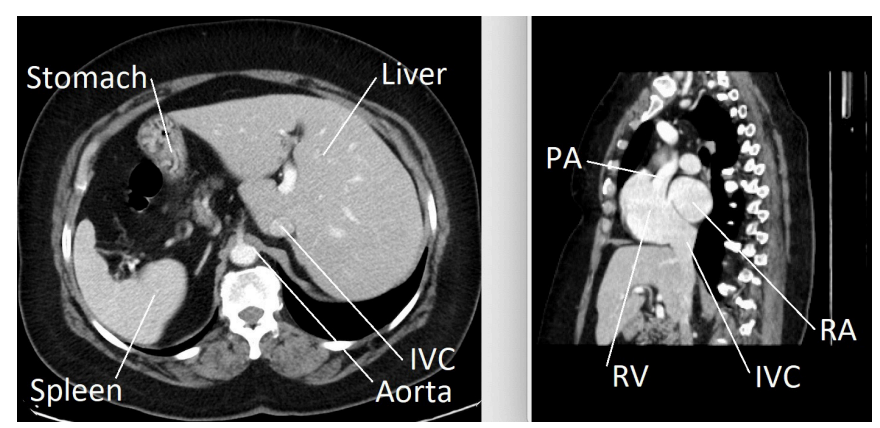

Figure 2. CT scan image of the great vessels showing that the main pulmonary artery is on the right of the ascending aorta, the liver is on the left, and the stomach and spleen are on the right side of the body, as expected in a patient with situs inversus totalis. IVC, inferior vena cava; PA, pulmonary artery; RV, right ventricle.

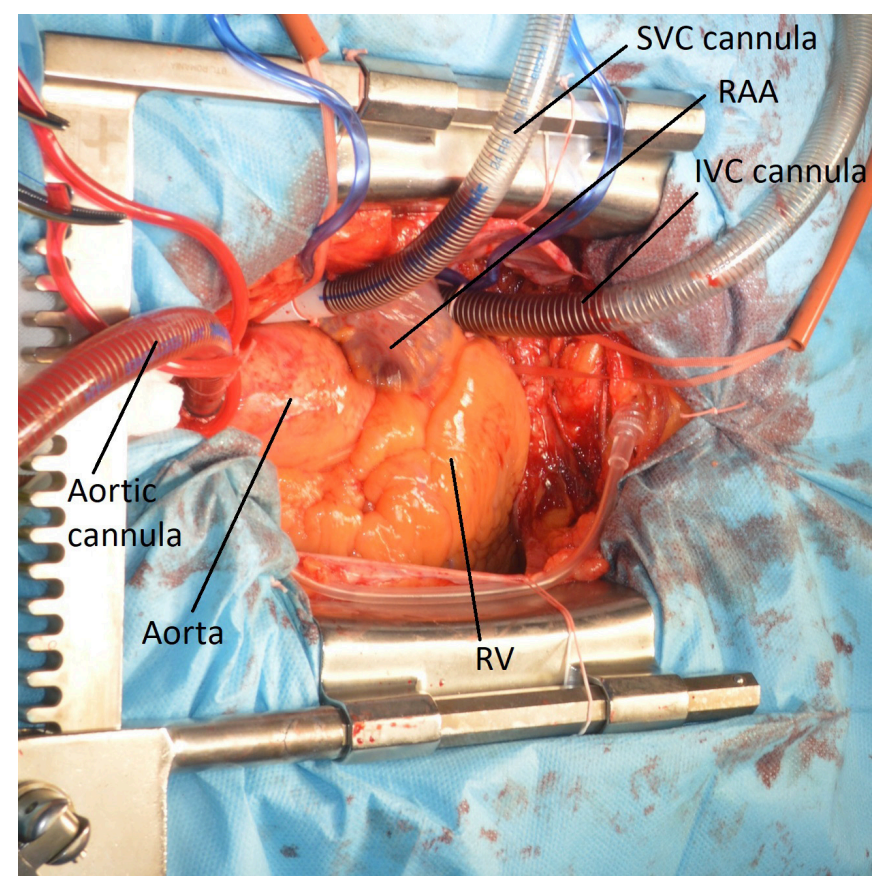

Figure 3. Intraoperative view showing the arterial and venous cannulation technique used. IVC, inferior vena cava; RAA, right atrial appendage; RV, right ventricle; SVC, superior vena cava.

showed that the heart was directed to the right with mild cardiomegaly. In childhood, the patient had been diagnosed with asymptomatic situs inversus totalis. Transesophageal echocardiography (TEE) was challenging: it confirmed severe mitral regurgitation with mild stenosis, but a different approach was needed to acquire the desired views by changing the position of the patient. The TEE 4-chamber and transgastric shortaxis views were obtained at a $0^{\circ}$ angle, having the left ventricle located on the left side of the TEE screen, which is the exact opposite of the normal TEE view (Figure 1). TEE revealed severe mitral valve regurgitation with an eccentric regurgitant jet that covered $>40 \%$ of the left atrium and vena contracta width of $0.7 \mathrm{~cm}$, normal left and right ventricle ejection

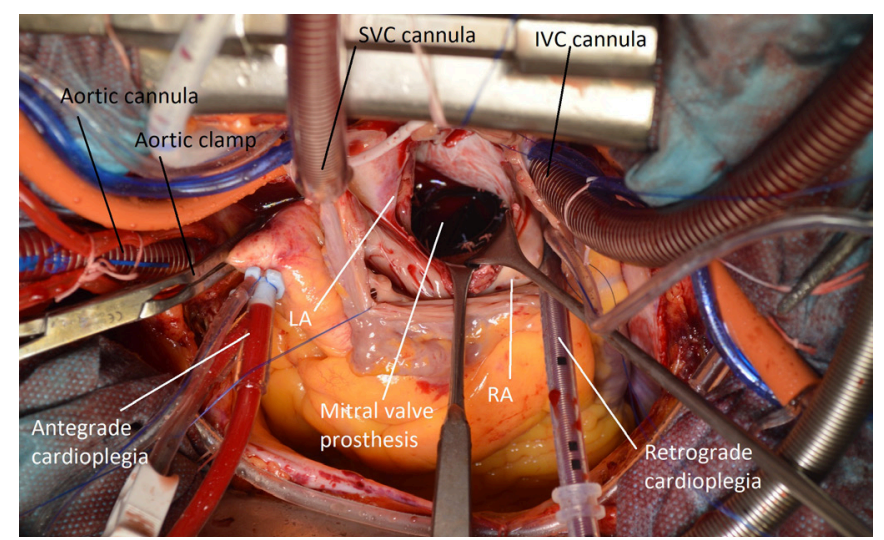

Figure 4. Intraoperative view showing the mechanical mitral valve prosthesis in situ. IVC, inferior vena cava; SVC, superior vena cava.

fractions, slightly enlarged dimensions of both ventricles, and mild left atrial enlargement. CT and abdominal tomography were performed to differentiate between situs inversus totalis and situs inversus solitus and determine whether there were any particularities of the inferior vena cava or in the placement of the abdominal organs. The CT scan showed the anatomic relations between the great vessels: the pulmonary artery trunk on the right of the ascending aorta (an inverted relationship, as expected in a patient with situs inversus totalis), normal continuity of the inferior vena cava, and absence of a third aberrant superior vena cava (Figure 2); all of these aspects are highly important to establish a good operative plan. Preoperative coronarography showed normal coronary arteries.

The patient was scheduled for elective mitral valve replacement surgery using a standard central normothermic cardiopulmonary bypass (CPB). After the median sternotomy and pericardiotomy were performed, we evaluated the anatomy of the heart and the great vessels and found a mirror image of a normal heart, the pulmonary artery on the right side of the ascending aorta, the right ventricle on the left and the left ventricle on the right, and normal insertion of both superior and inferior caval veins at the level of the right atrium. After complete inspection of the mediastinum and contents, the surgeon remained on the right side of the patient, and $\mathrm{CPB}$ was established by bicaval and ascending arterial cannulation (Figure 3). After the beginning of $\mathrm{CPB}$, the surgeon switched his position from the right to the left side of the patient. Myocardial protection was initiated with antegrade cold blood $\left(6^{\circ} \mathrm{C}\right.$ to $\left.8^{\circ} \mathrm{C}\right)$ cardioplegia (4 parts blood to 1 part St. Thomas's II cardioplegic solution) and was maintained with a single dose delivered in a retrograde manner after 20 minutes.

We decided to approach the mitral valve through a right atriotomy with an interatrial transseptal incision because of the posterior rotation and deep position of the left atrium. For that, it was necessary to isolate the right heart by encircling with snares both the superior and inferior venae cavae. After the heart was arrested, both caval snares were securely tightened in place. Right atriotomy ran parallel 
to the atrioventricular sulcus. A retrograde coronary sinus cardioplegia catheter (Edwards Lifesciences, Irvine, CA) was introduced in the coronary venous sinus and secured through a purse-string suture, and its position was verified by manual palpation and transesophageal echocardiography. We achieved optimal exposure of the mitral valve through an incision in the interatrial septum started at the level of the fossa ovalis, which ran parallel to the right atriotomy [Escobar 1997] and extended for approximately $5 \mathrm{~cm}$ in a cephalad direction toward the left atrial roof. Intraoperative evaluation of the mitral valve revealed an insufficient valve, with the posterior leaflet degenerated, calcified free margins, flail of the middle (A2) and medial (A3) segments of the anterior leaflet, elongated anterior chordae, and mitral valve annulus severely dilated, making it unsuitable for valve repair. Therefore, the native valve was excised, without preserving the calcified posterior leaflet, and replaced with a bileaflet mechanical valve prosthesis (Carbomedics no. 27; Carbomedics Inc., Austin, TX) (Figure 4). The interatrial septum incision and the right atriotomy were closed with double-layer continuous polypropylene 4-0 sutures.

The patient's postoperative evolution was uneventful, and we were able to discharge her from the hospital on the 7 th postoperative day. Postoperative chest X-ray was normal (Figure 5). Transthoracic postoperative echocardiography revealed a normally functioning mechanical prosthesis with a mean gradient of $5 \mathrm{mmHg}$ across the prosthesis and normal left and right ventricular function.

\section{DISCuSSION}

A patient presenting with dextrocardia associated with situs inversus totalis has the mirror-image organ placement form of the thoracic and abdominal viscera. To date, several abnormalities for dextrocardiac patients have been published. Uchimiro et al [2012] published the case of a patient with an interrupted inferior vena cava in the intrahepatic segment. In this patient, preoperative CT showed both dextrocardia and situs inversus, but also an interruption of the inferior vena cava continued with an azygos vein and direct drainage of the hepatic vein into the right atrium. Major intraoperative difficulties regarding these abnormalities are especially associated with inferior vena cava cannulation and appropriate venous drainage of the liver. In a similar case, supplementary venous cannulae were inserted into the hepatic vein from the right atrium [Koga 2019]. Therefore, CT is a useful tool for detecting abnormalities of the caval veins in all cases of dextrocardia. The left atrial approach and a single dual-stage venous cannula may be used in cases in which the right ventricle blocks access to the inferior vena cava [Mhonchan 2013]. In most cases, central cannulation is used, but in cases of situs inversus with posterior rotation and deep position of the left atrium, because of the malposition and poor accessibility of the inferior vena cava, many surgeons have preferred peripheral cannulation of the femoral vein. They support their decision by the need to lift the heart for decannulation of the inferior vena cava: doing this maneuver with a mitral valve prosthesis in situ increases the risk of a left ventricle rupture [St. Rammos 1996].

In our case, preoperative CT scan showed no abnormalities of the inferior and superior cava position at the level of the right atrium, and the dimensions of the right ventricle were almost normal, which allowed an easy dissection, placement of snares on the inferior vena cava, and optimal cannulation of the inferior vena cava. Although in other cases, the mitral valve has been accessed through the left atrium (75\%) [Berhane 2016], we believe that our approach by transseptal mitral replacement with bicaval cannulation strategy is a sure and reproducible technique that can also be used in patients with associated tricuspid valve disease. Thorough preoperative study and understanding of the anatomy of patients with dextrocardia associated with situs inversus is the key to successful mitral valve surgery.

\section{CONCLUSION}

A transseptal approach using an incision at the level of the fossa ovalis extended in a cephalad direction toward the left atrial roof direction in a patient with dextrocardia and situs inversus totalis has proved to be an excellent approach that ensures wide exposure of the mitral valve and no morbidity in the case of mitral valve replacement. Preoperative CT scan is mandatory to establish a specific operative plan and assess the presence of any anatomic abnormalities that may interfere in the cannulation strategy or the operative technique.

\section{DECLARATION OF CONFLICTING INTERESTS}

The authors declare no conflicts of interest with respect to the authorship and/or publication of this article.

\section{REFERENCES}

Escobar FS, Gallardo GS, Marroquin SR, Soriano FL, García RB. 1997. The transseptal approach for mitral valve replacement revisited. Tex Heart Inst J 24:209-214.

Garg N, Agarwal BL, Modi N, Radhakrishnan S, Sinha N. 2009. Dextrocardia: An analysis of cardiac structures in 125 patients. Int J Cardiol $88: 143-155$.

Kikon M, Kazmi A, Gupta A, Grover V. 2013. Left-sided approach for mitral valve replacement in a case of dextrocardia with situs solitus. Interact Cardiovasc Thorac Surg 17:900-902.

Koga S, Katayama Y, Koga K, Uchino M. 2019. Mitral valve and tricuspid valve regurgitation in a patient with dextrocardia and absence of the inferior vena cava [in Japanese]. Kyobu Geka 72:587-590.

Lowenthal A, Tacy T, Punn R. 2019. Heterotaxy (isomerism of the atrial appendages): Anatomy, clinical features, and diagnosis. UpToDate. Waltham, MA: UpToDate Inc. https://www.uptodate.com/contents/heterotaxy-isomerism-of-the-atrial-appendages-anatomy-clinical-featuresand-diagnosis. Accessed December 8, 2019.

Maldjian PD, Saric M. Approach to dextrocardia in adults: Review. 2007. 
AJR Am J Roentgenol 188(6 suppl):S39-S49.

St. Rammos K, Bakas AJ, Panagopoulos FG. 1996. Mitral valve replacement in Jehovah's Witness with dextrocardia and situs solitus. J Heart Valve Dis 5:673-674.

Uchimuro T, Fukui T, Matsuyama S, Tabata M, Takanashi S. 2012. Mitral valve replacement in dextrocardia and situs inversus. Kyobu Geka
65:858-861.

Worku B, Robinson S, Gulkarov I, Acinapura A, Tortolani AJ. 2016. Mitral valve operation in dextrocardia. Ann Thorac Surg 101:e161.

Zariwala MA, Knowles MR, Leigh, MW. 2019. Primary ciliary dyskinesia. GeneReviews https://www.ncbi.nlm.nih.gov/books/NBK1122/. Accessed December 8, 2019. 